\title{
Nanomedicine in Oncocardiology: Contribution and Perspectives of Preclinical Studies
}

\author{
Gabriel Silva Marques Borges ${ }^{1,2 t}$, Eduardo Burgarelli Lages ${ }^{1,2 t}$, Pierre Sicard ${ }^{2,3}$, \\ Lucas Antônio Miranda Ferreira ${ }^{1}$ and Sylvain Richard ${ }^{2,3 *}$
}

${ }^{1}$ Departamento de Produtos Farmacêuticos, Faculdade de Farmácia, Universidade Federal de Minas Gerais, Belo Horizonte, Brazil, ${ }^{2}$ PhyMedExp, Université de Montpellier, INSERM, CNRS, Montpellier, France, ${ }^{3}$ IPAM, BioCampus, CNRS, INSERM,

Université de Montpellier, Montpellier, France

OPEN ACCESS

Edited by:

Carlo Gabriele Tocchetti,

University of Naples Federico II, Italy

Reviewed by:

Fabiana Passaro,

University of Naples Federico II, Italy

Gianluca Testa,

University of Molise, Italy

*Correspondence:

Sylvain Richard

sylvain.richard@inserm.fr

tThese authors have contributed equally to this work and share first authorship

Specialty section:

This article was submitted to Cardio-Oncology,

a section of the journa

Frontiers in Cardiovascular Medicine

Received: 07 April 2021

Accepted: 01 June 2021

Published: 30 June 2021

Citation:

Borges GSM, Lages EB, Sicard P, Ferreira LAM and Richard S (2021) Nanomedicine in Oncocardiology: Contribution and Perspectives of Preclinical Studies.

Front. Cardiovasc. Med. 8:690533.

doi: 10.3389/fcvm.2021.690533
Cancer and cardiovascular diseases are the leading causes of death and morbidity worldwide. Strikingly, cardiovascular disorders are more common and more severe in cancer patients than in the general population, increasing incidence rates. In this context, it is vital to consider the anticancer efficacy of a treatment and the devastating heart complications it could potentially cause. Oncocardiology has emerged as a promising medical and scientific field addressing these aspects from different angles. Interestingly, nanomedicine appears to have great promise in reducing the cardiotoxicity of anticancer drugs, maintaining or even enhancing their efficacy. Several studies have shown the benefits of nanocarriers, although with some flaws when considering the concept of oncocardiology. Herein, we discuss how preclinical studies should be designed as closely as possible to clinical protocols, considering various parameters intrinsic to the animal models used and the experimental protocols. The sex and age of the animals, the size and location of the tumors, the doses of the nanoformulations administered, and the acute vs. the long-term effects of treatments are essential aspects. We also discuss the perspectives offered by non-invasive imaging techniques to simultaneously assess both the anticancer effects of treatment and its potential impact on the heart. The overall objective is to accelerate the development and validation of nanoformulations through high-quality preclinical studies reproducing the clinical conditions.

Keywords: anticancer drugs, cardiotoxicity, nanoformulations, non-invasive imaging, echography, photoacoustic, small animals

\section{INTRODUCTION}

Recent advances in the treatment of cancers have improved patient care and cure rates. Cancer, once fatal, is now emerging as a chronic disease, often at the cost of cardiovascular complications. Moreover, cardiovascular diseases (CVD) are more common and more severe in cancer patients (1-5). In this context, the development of novel treatments with antineoplastic agents still raises concerns about undesirable effects at the acute phase of the treatment and potentially during long-term therapy (6). Therefore, the management of cancer patients has moved from general cardiology to a specialized discipline, oncocardiology, with in-depth cardiovascular monitoring at each stage of cancer therapy $(1,7)$. Among various areas that can help solve these problems, nanotechnology is being increasingly investigated in preclinical studies to improve anticancer treatments and reduce 
their cardiotoxicity. This review will discuss the potential of nanocarriers to optimize the use of anticancer drugs through tailored preclinical approaches that can help understand/control their adverse effects and how preclinical models can be better designed to represent an oncocardiology approach truly.

\section{REDUCING CARDIOTOXICITY OF ANTICANCER DRUGS THROUGH NANOTECHNOLOGY}

Cardiotoxicity is a broad term that embraces toxic effects on cardiac structure, morphology, rest parameters, and the dynamic function of the heart. Cytotoxic drugs and targeted therapies used to treat cancer, including classic chemotherapeutic agents, tyrosine kinase inhibitors, antiangiogenic drugs, and immunotherapy, often impair the cardiovascular system (8). Of note, cancer itself may promote CVD (6).

The cardiotoxicity evoked by conventional chemotherapy is widely known and occur through multiple mechanisms, including overproduction of free radical species, inhibition of topoisomerase $2 \beta$ causing DNA double-strand breaks and activation of apoptosis, profound changes in the transcriptome leading to the generation of reactive oxygen species, and inhibition of VEGF receptors causing endothelial dysfunction and vascular injury. In turn, the novel immunotherapeutic agents can lead to the inhibition of the Human Epithelial Receptor type 2 (HER2), impairing the cell signaling in cardiomyocytes; and the inhibition of programmed cell death proteins (PD-1) causing inflammatory infiltrates in the heart tissue $(8-10)$. This cardiotoxicity can lead to bradycardia (paclitaxel and thalidomide), promote QT interval prolongation and arrhythmias (amsacrine and anthracyclines), evoke myocardial ischemia via coronary vasospasm (antimetabolites and 5-fluorouracil), impair the left ventricle function (anthracyclines, tyrosine kinase inhibitors, alkylating agents, and cisplatin), and evoke myocarditis, pericarditis, and heart failure (immunotherapy) $(6,11,12)$.

The objective of cardioprotection is to limit cardiac damage while maintaining antineoplastic efficacy (8). In the clinics, combinations with drugs like statins, antiarrhythmic, betablockers, calcium-channel inhibitors, and ACE (angiotensinconverting enzyme) inhibitors are some of the strategies (13). Interestingly, preclinical and clinical studies show that nanotechnology can also lead to cardioprotection in cancer treatment (14).

The basic concept of nanomedicine is to alter both pharmacokinetics and biodistribution of nanoencapsulated drugs, increasing their accumulation in the tumor site and decreasing their delivery to non-target organs, such as the heart. Passive and active targeting mechanisms can enable nanocarriers to reach the tumor site efficiently. The passive mechanism is based on the Enhanced Permeability and Retention (EPR) effect, which leads to the accumulation of nanostructures in the tumor site due to the characteristic leaky vasculature and absence of lymphatic drainage in the tumor microenvironment (Figure 1A)
(15). In turn, active targeting involves the attachment of highaffinity ligands to tumor cells on the surface of the nanocarriers. The idea is to use ligands for which cancer cells express a high number of specific receptors, whereas normal cells express very few. A wide variety of ligands have been used for this purpose, including folic acid, hyaluronic acid, transferrin, among others (Figure 1B) $(15,16)$. Another interesting approach to evoke cardioprotection through nanotechnology is the co-delivery of antineoplastic drugs and cardioprotective agents (17). For example, the co-encapsulation of DOX with curcumin, quercetin, or docosahexaenoic acid within the same nanocarrier has been proposed in several nanoscale systems (18-20).

In 1995, Doxil ${ }^{\circledR}$ (pegylated liposomal DOX) was the first nanocarrier approved for clinical use $(21,22)$. This formulation was designed to reduce DOX toxicity while preserving its antitumor efficacy by altering its tissue distribution and pharmacokinetics (23). Several randomized controlled trials have demonstrated a reduction in the risk of clinical cardiotoxicity for liposomal DOX-based chemotherapy compared to free DOX (24, 25). At present, various commercial formulations of nanocarriers have been approved or are under clinical trial for cancer therapy. These nanocarriers deliver classic chemotherapeutics, such as isolated paclitaxel, irinotecan, the synergistic combination of daunorubicin and cytarabine, and recent innovative treatments for T-cell cancer immunotherapy $(26,27)$.

\section{CONSIDERATIONS ABOUT PRECLINICAL STUDIES OF NANOCARRIERS}

The evaluation of the cardiotoxicity of nanostructures can be performed both in vitro and in vivo. Human pluripotent stem cell-derived cardiomyocytes (hiPSC-CM) emerge as a practical approach for the early in vitro screening of cardiotoxicity of new drugs (28-30). However, investigations on animal models are still required to develop their use in clinical practice despite some limitations. Nonetheless, some characteristics of these models, such as age, gender, drug doses, and tumor models, are often not correctly considered (Figure 2A). We will discuss these aspects in the following sections.

\section{Age and Gender}

Most cancer patients are more than 60 years old, with aging being a critical predisposition factor for cancer and CVD (5). Despite these predictions, many preclinical cancer studies use mice whose age correlates with that of adolescents (Figure 2A), a period of life when cancer and cardiovascular events are fortunately rare. The physiological differences between young and old animals are significant, and one example is related to the EPR effect. Tumor-bearing young animals may exhibit a more pronounced EPR effect than older animals since older subjects present a lower tumor angiogenesis rate (31). This issue makes less probable the formation of blood vessels with fenestrations allowing the higher permeation of nanocarriers by tumor tissues in young animals compared to older subjects (32). Cardiovascular adverse effects of therapeutics can also be underestimated in young animals since the hearts of these animals have a higher regenerative potential 


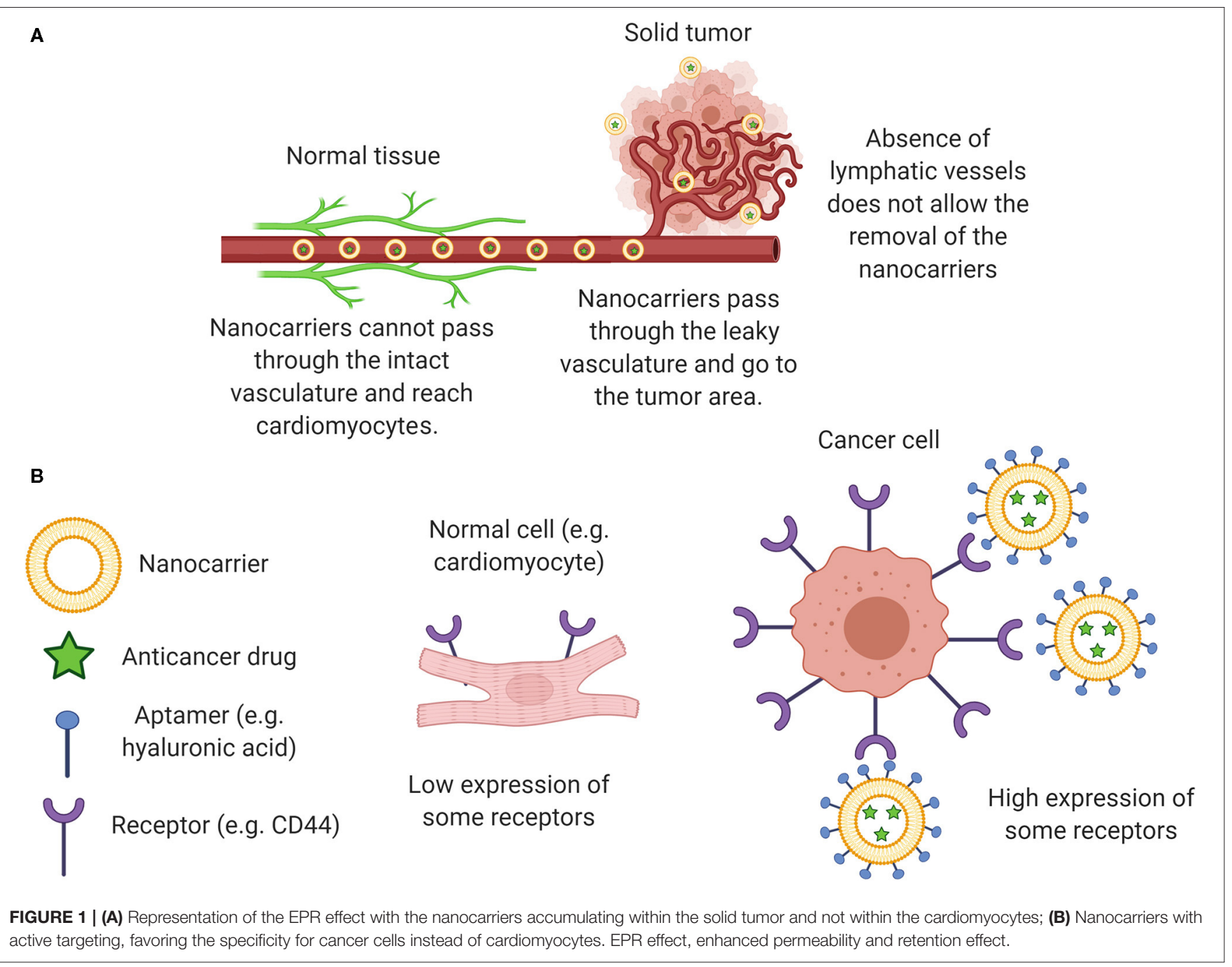

than the hearts of old animals $(33,34)$. Therefore, the widespread use of very young animals is a serious flaw that impairs the translation of the results from preclinical to clinical studies.

Regarding gender, men are the most affected by almost all types of cancer, with a few exceptions, such as anorectal, gallbladder, thyroid, and breast cancer. However, we expect the number of women affected by neoplasms to increase. Therefore, it is essential to evaluate some risk factors affecting women when they are gender-specific or affect women more intensely, such as menopause, smoking, diabetes, and pregnancy (35). Additionally, drug treatments for CVD may not be the same due to physiological differences between the sexes (36). As an example, anthracyclines-induced cardiotoxicity can be less pronounced in women due to some benefits provided by estrogens (37). Despite all this, most studies use only male animals (Figure 2A), which does not reveal whether a particular cancer treatment works in the same way in women or whether its side effects are the same. Overall, preclinical cancer research should include sex as a biological variable in all investigations (35) (Figure 2B).

\section{Tumor Models}

Regarding the formation of tumors in animal models, there are issues such as their location and size. The orthotopic localization provides the appropriate microenvironment to mimic metastases caused by a specific tumor (38). However, the establishment of orthotopic tumors is an exception, and most studies involve subcutaneous inoculation of tumor cells in the flank of the animals. In addition, the sizes of the tumors implanted subcutaneously are generally substantial to allow their measurements through calipers. Consequently, the relationship between tumor weight/animal weight becomes much higher than that observed in humans, leading to errors when interpreting the results. Human tumors treated with nanomedicine are usually in the order of a few grams, so the tumor/body weight relationship is insignificant. In mice, on the other hand, tumors can reach up to $10 \%$ of the animal's weight. Large size is one factor that causes the EPR effect of nanomedicines to be overestimated in animals, as larger tumors can promote higher colloidal buildup (39). Ideally, the tumors should not exceed few millimeters to respect better the relationship between tumor and body weight 
A

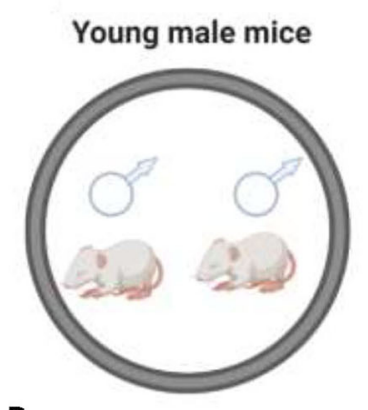

B

\section{Adult male/female mice}

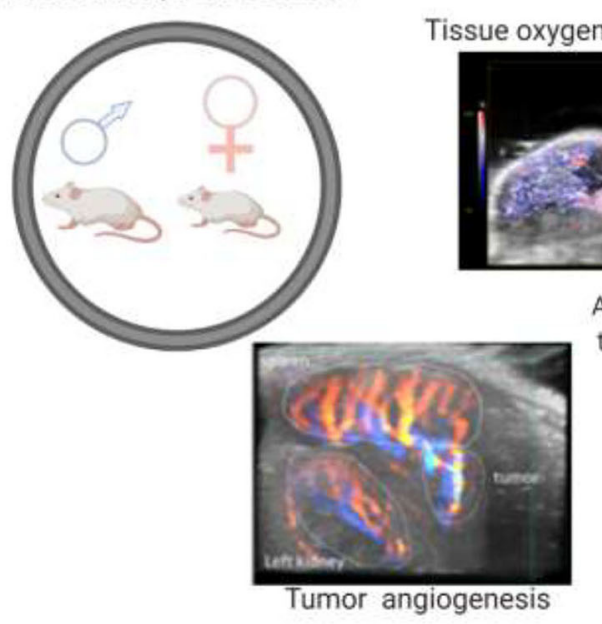

Short period of treatment with high doses Evaluation of cardiovascular health post mortem

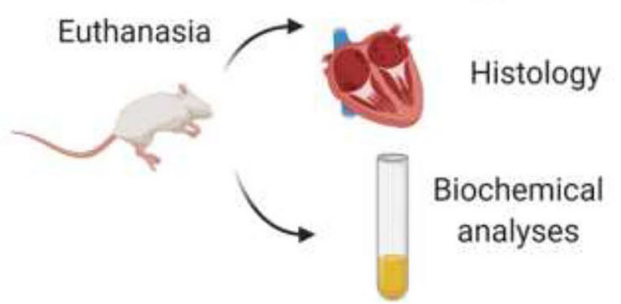

Longer period of treatment with adequate doses Evaluation of cardiovascular health during and after the treatment

issue oxygen saturation level

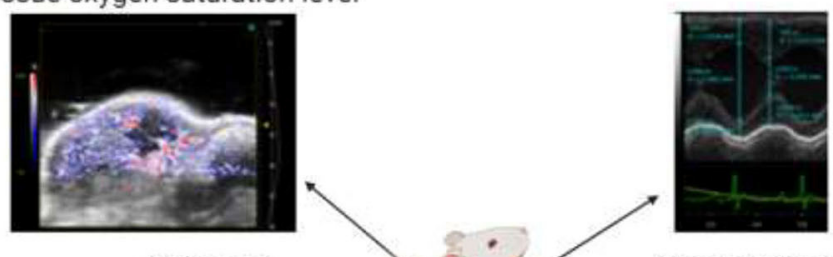

Non invasive assessment of cardiac morphology and function by echocardiography

$(2 \mathrm{D}, 3 \mathrm{D}$, speckle tracking, ...)

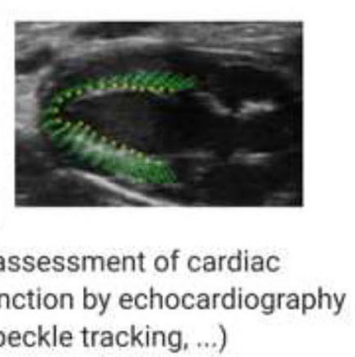

Detection of small

tumor volumes and metastasis foci

FIGURE 2 | (A) Representation of how anticancer animal studies are generally conducted up to now, with cardiotoxicity evaluation being conducted only after euthanasia of the animals, with high doses administered of the nanocarriers and the use of only male and very young animals; (B) Suggestion of how to conduct animal studies with nanocarriers using both female and male older mice; evaluation of cardiotoxicity during and after the treatment administered with lower doses of the nanocarriers. The ultrasound-based techniques allow the simultaneous evaluation of antitumor activity and cardiotoxicity.

observed in humans. Additionally, small tumors allow more prolonged treatment and animal monitoring periods, as animals must be euthanized when the tumors reach high volumes.

\section{Administered Doses of Nanomedicines}

It is essential to administer, to the animals, doses related to the plasma blood concentrations found in the clinics (40). However, the choice of dosage in efficacy and toxicity preclinical studies is often neglected, compromising the success of these therapies in humans. First, very high doses are often utilized in animal studies to reduce large volume tumors. Therefore, the use of lower doses relies on the inoculation of smaller volume tumors in the animals. In addition, the maximum tolerated doses in mice are generally higher than in humans (40). Thus, the doses used in animal models can sometimes not be applied to human studies, making the clinical translation of these formulations limited. In the specific case of nanocarriers, their reduced systemic toxicity allows the administration of even higher doses to the animals (41). Nonetheless, one should stress that reduced acute toxicity does not always mean reduced chronic toxicity $(42,43)$.

\section{Evaluation of Chronic Cardiotoxic Events}

An important concept to apply in preclinical studies with nanocarriers is the follow-up of animals after anticancer treatment. Many significant cardiac side effects often occur later, even years after antineoplastic therapy (44). However, animals are often euthanized rapidly after the treatment is completed (39). Additionally, most preclinical studies using nanotechnology for the treatment of cancer only perform postmortem cardiovascular assessments. These analyses are based mainly on the biochemical quantification of certain enzymes and the histology of the heart. Although the information obtained by these methods is valid, this does not reflect an oncocardiology approach, as they do not monitor cardiovascular health during cancer treatment, as practiced in human patients. Moreover, studies that focus on cardiovascular toxicity are usually done in healthy animals rather than in animals with tumors, with some drawbacks because the toxic effects may differ in non-tumor subjects and tumor carriers (45).

The following of laboratory animals through more extensive periods, although desirable, can be costly and laborious for 
the researchers. One exciting option is the use of domestic animals such as dogs and cats. These species spontaneously develop tumors, and the owners maintain their pets through the years after the diagnosis. These animals can participate in experimental therapies, close to clinical trials, evaluating tumor regression and cardiovascular health in partnership with veterinaries $(46,47)$.

\section{Simultaneous Evaluation of Antitumor and Cardiotoxic Effects}

Anticancer activity and cardiotoxicity should, when possible, be considered simultaneously (Figure 2B). A holistic approach could help in the exploitation of divergent results. For example, the combination of DOX, trastuzumab, and taxanes is very effective in treating breast cancer, but it produces severe synergistic cardiotoxic events in female patients $(48,49)$. Additionally, premature aging induced by cancer treatment may contribute to chronic health problems in cancer survivors (7). Moreover, the nanocarriers can evoke cardiotoxicity per se. One example is the immune reactions to polyethylene glycol molecules attached to the surface of nanocarriers, known as complement activation-related pseudoallergy, which can even lead to a patient's death due to arrhythmias, ventricular fibrillation, and cardiac arrest (50). Moreover, oncolytic viruses, an increasingly used drug delivery vehicle, can replicate themselves in non-targeted tissues, evoking inflammation of the heart, leading to myocarditis (51).

Few preclinical studies have monitored both antitumor activity and cardiac function, in the same animal model, during and after the treatment with nanocarriers. To the best of our knowledge, the three studies presented below are the only ones to have evaluated both simultaneously, all of them using DOX-loaded nanocarriers. DOX loaded in folatecoated liposomes, injected intravenously in breast tumor-bearing mice, showed higher antitumor activity than free DOX and reduced cardiotoxicity (52). Similar findings were found after administering a liposomal formulation loading DOX to colon tumor-bearing mice (53). In addition, polymeric magnetic nanoparticles co-encapsulating DOX and verapamil (a calcium channel blocker) with active targeting of RGD (arginyl glycyl aspartic acid) peptide improved antitumor activity and decreased cardiotoxicity compared to free DOX (54). All these studies evaluated cardiotoxicity during treatment by analysis of the ECG and the QT interval. Imaging techniques have not been used, although they can provide essential data. To monitor smaller and orthotopic tumors and cardiotoxicity more precisely, during and after treatment, the use of non-invasive imaging techniques should be encouraged (Figure 2B).

\section{Ultrasound Techniques to Assess the Antitumor Activity and Cardiotoxicity of Nanomedicines}

Different imaging techniques such as magnetic resonance imaging (MRI), positron emission tomography (PET), SPECT (single photon emission tomography), and computed tomography (X - computer tomography) can simultaneously assess antitumor activity and cardiotoxicity evoked by anticancer treatments (55-57). These imaging techniques allow the early detection and monitoring of a wide range of cardiotoxic events, including systolic and diastolic dysfunction, morphological changes, coronary artery, valve, and pericardial diseases resulting from cancer treatment (56). However, the significant problems with these techniques are their high cost, long scanning time (30-60 min/animal), and radiation exposure (PET, SPECT, and CT) (58).

Real-time image acquisition, ease of data interpretation, and low cost make ultrasound imaging a promising and suitable approach, compared to other techniques, to monitor cardiotoxic events in preclinical studies (59-63). Ultrasound allows rapid longitudinal monitoring $(<10 \mathrm{~min} /$ animal for each control) of tumor and cardiac function before, during, and after anticancer treatment. This opportunity is of particular interest for monitoring tumor growth, as it is possible to monitor the animals for more extended periods $(64,65)$ and to assess the volume of implanted orthotopic tumors (66). This approach also makes it possible, on a practical level, to homogenize the different study groups as a function of a reference tumor volume. This possibility reduces the number of animals used while minimizing the variability in the distribution within each experimental group.

Finally, photoacoustic imaging (PAI) is another promising ultrasound-based technique. It is a relatively new hybrid modality that combines optical imaging contrast with the spatial resolution of ultrasound $(67,68)$. PAI provides high-resolution images of optical absorption in deep tissues, allowing visualization of angiogenesis, tissue oxygen saturation, and metabolic or inflammatory parameters (69-71). In addition, PAI has shown promise in detecting, diagnosing, and guiding cancer treatment due to its ability to detect or activate specific nanostructures to enhance contrast $(72,73)$.

\section{CONCLUDING REMARKS}

Clinics have widely demonstrated the concept that nanomedicines improve the therapeutic index of anticancer drugs $(15,74)$. The advances in nanoscale delivery systems offer great hope to increase the efficacy of drugs in a targeted manner and overcome the toxicity limitations associated with conventional free drug delivery. Translating the results from preclinical studies to more clinically relevant models is an urgent demand and a significant challenge to alleviate the burden of the undesirable cardiotoxic effects of anticancer drugs $(41,75)$. Considering the advantages and limitations of animal models and the peculiarities of nanoformulations, appropriate techniques for monitoring antitumor efficacy and cardiovascular health will be essential to practice the best oncocardiology approach.

\section{AUTHOR CONTRIBUTIONS}

All authors contributed to the writing and editing of the manuscript. 


\section{FUNDING}

GB and EL thank CAPES (Coordenação de Aperfeiçoamento de Pessoal de Nível Superior, Brazil) and FAPEMIG (Fundação de Amparo à Pesquisa do Estado de Minas Gerais, Brazil) for the scolarhips provided. SR, PS, and LF acknowledge CAPESCOFECUB for supporting a bilateral research collaboration

\section{REFERENCES}

1. Cautela J, Lalevée N, Ammar C, Ederhy S, Peyrol M, Debourdeau P, et al. Management and research in cancer treatment-related cardiovascular toxicity: challenges and perspectives. Int J Cardiol. (2016) 224:366-75. doi: 10.1016/j.ijcard.2016.09.046

2. Curigliano G, Cardinale D, Dent S, Criscitiello C, Aseyev O, Lenihan D, et al. Cardiotoxicity of anticancer treatments: epidemiology, detection, and management. CA Cancer J Clin. (2016) 66:309-25. doi: 10.3322/caac.21341

3. Masoudkabir F, Sarrafzadegan N, Gotay C, Ignaszewski A, Krahn AD, Davis MK, et al. Cardiovascular disease and cancer: evidence for shared disease pathways and pharmacologic prevention. Atherosclerosis. (2017) 263:343-51. doi: 10.1016/j.atherosclerosis.2017.06.001

4. Moslehi J, Fujiwara K, Guzik T. Cardio-oncology: a novel platform for basic and translational cardiovascular investigation driven by clinical need. Cardiovasc Res. (2019) 115:819-23. doi: 10.1093/cvr/cvz048

5. Okura Y, Takayama T, Ozaki K, Tanaka H, Kikuchi A, Saito T, et al. Future projection of cancer patients with cardiovascular disease in Japan by the year 2039: a pilot study. Int J Clin Oncol. (2019) 24:983-94. doi: 10.1007/s10147-019-01426-w

6. Lenneman Carrie G., Sawyer Douglas B. Cardio-oncology. Circ Res. (2016) 118:1008-20. doi: 10.1161/CIRCRESAHA.115.303633

7. Abe J-I, Martin JF, Yeh ETH. The future of onco-cardiology: we are not just "Side Effect Hunters." Circ Res. (2016) 119:896-9. doi: 10.1161/CIRCRESAHA.116.309573

8. Witteles RM, Bosch X. Myocardial protection during cardiotoxic chemotherapy. Circulation. (2015) 132:1835-45. doi: 10.1161/CIRCULATIONAHA.114.010486

9. Yeh ETH, Chang H-M. Oncocardiology-past, present, and future: a review. JAMA Cardiol. (2016) 1:1066-72. doi: 10.1001/jamacardio.2016.2132

10. Manolis AA, Manolis TA, Mikhailidis DP, Manolis AS. Cardiovascular safety of oncologic agents: a double-edged sword even in the era of targeted therapies - Part 2. Expert Opin Drug Saf. (2018) 17:893-915. doi: 10.1080/14740338.2018.1513489

11. Bansal N, Amdani S, Lipshultz ER, Lipshultz SE. Chemotherapy-induced cardiotoxicity in children. Expert Opin Drug Metab Toxicol. (2017) 13:817-32. doi: 10.1080/17425255.2017.1351547

12. Ganatra S, Parikh R, Neilan TG. Cardiotoxicity of immune therapy. Cardiol Clin. (2019) 37:385-97. doi: 10.1016/j.ccl.2019.07.008

13. Herrmann J. Adverse cardiac effects of cancer therapies: cardiotoxicity and arrhythmia. Nat Rev Cardiol. (2020) 17:474-502. doi: 10.1038/s41569-020-0348-1

14. Monahan DS, Almas T, Wyile R, Cheema FH, Duffy GP, Hameed A. Towards the use of localised delivery strategies to counteract cancer therapy-induced cardiotoxicities. Drug Deliv Transl Res. (2021) doi: 10.1007/s13346-020-00885-3. [Epub ahead of print].

15. Wicki A, Witzigmann D, Balasubramanian V, Huwyler J. Nanomedicine in cancer therapy: challenges, opportunities, and clinical applications. J Control Release. (2015) 200:138-57. doi: 10.1016/j.jconrel.2014.12.030

16. Tran S, DeGiovanni P-J, Piel B, Rai P. Cancer nanomedicine: a review of recent success in drug delivery. Clin Transl Med. (2017) 6:44. doi: 10.1186/s40169-017-0175-0

17. Yan T, Li D, Li J, Cheng F, Cheng J, Huang Y, et al. Effective codelivery of doxorubicin and curcumin using a glycyrrhetinic acid-modified chitosan-cystamine-poly( $\varepsilon$-caprolactone) copolymer micelle for combination cancer chemotherapy. Colloids Surf B Biointerfaces. (2016) 145:526-38. doi: $10.1016 /$ j.colsurfb.2016.05.070 program (no. 978/20) between Brazil (UFMG; Prof. Mônica Cristina De Oliveira) and France (Université de Montpellier; SR).

\section{ACKNOWLEDGMENTS}

The figures of this manuscript were made with the aid of Biorender.com.
18. Tahover E, Patil YP, Gabizon AA. Emerging delivery systems to reduce doxorubicin cardiotoxicity and improve therapeutic index: focus on liposomes. Anticancer Drugs. (2015) 26:241-58. doi: 10.1097/CAD.0000000000000182

19. Dou X-Q, Wang H, Zhang J, Wang F, Xu G-L, Xu C-C, et al. Aptamer-drug conjugate: targeted delivery of doxorubicin in a HER3 aptamer-functionalized liposomal delivery system reduces cardiotoxicity. Int J Nanomedicine. (2018) 13:763-76. doi: 10.2147/IJN.S149887

20. Lages EB, Fernandes RS, Silva J de O, de Souza ÂM, Cassali GD, de Barros ALB, et al. Co-delivery of doxorubicin, docosahexaenoic acid, and $\alpha$ tocopherol succinate by nanostructured lipid carriers has a synergistic effect to enhance antitumor activity and reduce toxicity. Biomed Pharmacother. (2020) 132:110876. doi: 10.1016/j.biopha.2020.110876

21. Okada H, Toguchi $H$. Biodegradable microspheres in drug delivery. Crit Rev Ther Drug Carrier Syst. (1995) 12:1-99. doi: 10.1615/critrevtherdrugcarriersyst.v12.i1.10

22. Park J, Fong PM, Lu J, Russell KS, Booth CJ, Saltzman WM, et al. PEGylated PLGA nanoparticles for the improved delivery of doxorubicin. Nanomedicine. (2009) 5:410-8. doi: 10.1016/j.nano.2009.02.002

23. Barenholz Y. Doxil $\AA-$ the first FDA-approved nano-drug: lessons learned. J Control Release. (2012) 160:117-34. doi: 10.1016/j.jconrel.2012. 03.020

24. Rafiyath SM, Rasul M, Lee B, Wei G, Lamba G, Liu D. Comparison of safety and toxicity of liposomal doxorubicin vs. conventional anthracyclines: a meta-analysis. Exp Hematol Oncol. (2012) 1:10. doi: 10.1186/2162-3619-1-10

25. Xing M, Yan F, Yu S, Shen P. Efficacy and cardiotoxicity of liposomal doxorubicin-based chemotherapy in advanced breast cancer: a meta-analysis of ten randomized controlled trials. PLoS ONE. (2015) 10:e0133569. doi: 10.1371/journal.pone. 0133569

26. Anselmo AC, Mitragotri S. Nanoparticles in the clinic: an update. Bioeng Transl Med. (2019) 4:e10143. doi: 10.1002/btm2.10143

27. Gong N, Sheppard NC, Billingsley MM, June CH, Mitchell MJ. Nanomaterials for T-cell cancer immunotherapy. Nat Nanotechnol. (2021) 16:25-36. doi: 10.1038/s41565-020-00822-y

28. Golforoush PA, Narasimhan P, Chaves-Guerrero PP, Lawrence E, Newton G, Yan R, et al. Selective protection of human cardiomyocytes from anthracycline cardiotoxicity by small molecule inhibitors of MAP4K4. Sci Rep. (2020) 10:12060. doi: 10.1038/s41598-020-68907-1

29. Lyra-Leite DM, Burridge PW. Pluripotent stem cell modeling of anticancer therapy-induced cardiotoxicity. Curr Cardiol Rep. (2020) 22:56. doi: 10.1007/s11886-020-01325-x

30. Schwach V, Slaats RH, Passier R. Human pluripotent stem cell-derived cardiomyocytes for assessment of anticancer drug-induced cardiotoxicity. Front Cardiovasc Med. (2020) 7:50. doi: 10.3389/fcvm.2020.00050

31. Bojovic B, Crowe DL. Chronologic aging decreases tumor angiogenesis and metastasis in a mouse model of head and neck cancer. Int J Oncol. (2010) 36:715-23. doi: 10.3892/ijo_00000547

32. Andriyanov AV, Portnoy E, Koren E, Inesa S, Eyal S, Goldberg $\mathrm{SN}$, et al. Therapeutic efficacy of combined PEGylated liposomal doxorubicin and radiofrequency ablation: comparing single and combined therapy in young and old mice. J Control Release. (2017) 257:2-9. doi: 10.1016/j.jconrel.2017.02.018

33. Dai D-F, Rabinovitch PS. Cardiac aging in mice and humans: the role of mitochondrial oxidative stress. Trends Cardiovasc Med. (2009) 19:213-20. doi: 10.1016/j.tcm.2009.12.004

34. Chiao YA, Rabinovitch PS. The aging heart. Cold Spring Harb Perspect Med. (2015) 5:a025148. doi: 10.1101/cshperspect.a025148 
35. Rubin JB, Lagas JS, Broestl L, Sponagel J, Rockwell N, Rhee G, et al. Sex differences in cancer mechanisms. Biol Sex Differ. (2020) 11:17. doi: 10.1186/s13293-020-00291-x

36. Maas AHEM, Appelman YEA. Gender differences in coronary heart disease. Neth Heart J. (2010) 18:598-602. doi: 10.1007/s12471-010-0841-y

37. Cadeddu Dessalvi C, Pepe A, Penna C, Gimelli A, Madonna R, Mele D, et al. Sex differences in anthracycline-induced cardiotoxicity: the benefits of estrogens. Heart Fail Rev. (2019) 24:915-25. doi: 10.1007/s10741-019-09 820-2

38. Lwin TM, Hoffman RM, Bouvet M. Advantages of patient-derived orthotopic mouse models and genetic reporters for developing fluorescenceguided surgery. J Surg Oncol. (2018) 118:253-64. doi: 10.1002/jso. 25150

39. Nichols JW, Bae YH. EPR: evidence and fallacy. J Control Release. (2014) 190:451-64. doi: 10.1016/j.jconrel.2014.03.057

40. Day C-P, Merlino G, Van Dyke T. Preclinical mouse cancer models: a maze of opportunities and challenges. Cell. (2015) 163:39-53. doi: 10.1016/j.cell.2015.08.068

41. Hare JI, Lammers T, Ashford MB, Puri S, Storm G, Barry ST. Challenges and strategies in anticancer nanomedicine development: an industry perspective. Adv Drug Deliv Rev. (2017) 108:25-38. doi: 10.1016/j.addr.2016.04.025

42. Yemm KE, Alwan LM, Malik AB, Salazar LG. Renal toxicity with liposomal doxorubicin in metastatic breast cancer. J Oncol Pharm Pract. (2019) 25:173842. doi: $10.1177 / 1078155218798157$

43. Savani M, Woerner K, Bu L, Birkenbach M, Skubitz KM. Pegylated liposomal doxorubicin-induced renal toxicity in retroperitoneal liposarcoma: a case report and literature review. Cancer Chemother Pharmacol. (2021) 87:289-94. doi: $10.1007 / \mathrm{s} 00280-020-04203-\mathrm{Z}$

44. Dong J, Chen H. Cardiotoxicity of anticancer therapeutics. Front Cardiovasc Med. (2018) 5:9. doi: 10.3389/fcrm.2018.00009

45. Wang F, Iskra B, Kleinerman E, Alvarez-Florez C, Andrews T, Shaw A, et al. Aerobic exercise during early murine doxorubicin exposure mitigates cardiac toxicity. J Pediatr Hematol Oncol. (2018) 40:208-15. doi: 10.1097/MPH.0000000000001112

46. Khanna C, Lindblad-Toh K, Vail D, London C, Bergman P, Barber L, et al. The $\operatorname{dog}$ as a cancer model. Nat Biotechnol. (2006) 24:1065-6. doi: 10.1038/nbt0906-1065b

47. Rossa C, D'Silva NJ. Non-murine models to investigate tumor-immune interactions in head and neck cancer. Oncogene. (2019) 38:4902-14. doi: 10.1038/s41388-019-0776-8

48. Gianni L, Salvatorelli E, Minotti G. Anthracycline cardiotoxicity in breast cancer patients: synergism with trastuzumab and taxanes. Cardiovasc Toxicol. (2007) 7:67-71. doi: 10.1007/s12012-007-0013-5

49. El-Sherbeny WS, Sabry NM, Sharbay RM. Prediction of trastuzumabinduced cardiotoxicity in breast cancer patients receiving anthracycline-based chemotherapy. J Echocardiogr. (2019) 17:76-83. doi: 10.1007/s12574-018-0394-4

50. Szebeni J. Complement activation-related pseudoallergy: a stress reaction in blood triggered by nanomedicines and biologicals. Mol Immunol. (2014) 61:163-73. doi: 10.1016/j.molimm.2014.06.038

51. Hazini A, Pryshliak M, Brückner V, Klingel K, Sauter M, Pinkert S, et al. Heparan sulfate binding coxsackievirus B3 strain PD: a novel avirulent oncolytic agent against human colorectal carcinoma. Hum Gene Ther. (2018) 29:1301-14. doi: 10.1089/hum.2018.036

52. de Oliveira Silva J, Fernandes RS, Ramos Oda CM, Ferreira TH, Machado Botelho AF, Martins Melo M, et al. Folate-coated, long-circulating and $\mathrm{pH}$-sensitive liposomes enhance doxorubicin antitumor effect in a breast cancer animal model. Biomed Pharmacother. (2019) 118:109323. doi: 10.1016/j.biopha.2019.109323

53. Razavi-Azarkhiavi K, Jafarian AH, Abnous K, Razavi BM, Shirani K, Zeinali $\mathrm{M}$, et al. The comparison of biodistribution, efficacy and toxicity of two PEGylated liposomal doxorubicin formulations in mice bearing c-26 colon carcinoma: a preclinical study. Drug Res. (2016) 66:330-6. doi: 10.1055/s-0035-1569447

54. Shen J-M, Gao F-Y, Yin T, Zhang H-X, Ma M, Yang Y-J, et al. cRGDfunctionalized polymeric magnetic nanoparticles as a dual-drug delivery system for safe targeted cancer therapy. Pharmacol Res. (2013) 70:102-15. doi: 10.1016/j.phrs.2013.01.009
55. Thuny F, Huttin O, Ederhy S. Cardio-oncology: Clinical and imaging perspectives for optimal cardiodetection and cardioprotection in patients with cancer. Arch Cardiovasc Dis. (2019) 112:550-8. doi: 10.1016/j.acvd.2019.07.001

56. Biersmith MA, Tong MS, Guha A, Simonetti OP, Addison D. Multimodality cardiac imaging in the era of emerging cancer therapies. J Am Heart Assoc. (2020) 9:e013755. doi: 10.1161/JAHA.119.013755

57. López-Fernández T. Cardiac imaging in oncology patients in Europe: a model for advancement of CV safety and development of comprehensive CV care. J Cardiovasc Transl Res. (2020) 13:490-4. doi: 10.1007/s12265-020-10028-1

58. Moran CM, Thomson AJW, Rog-Zielinska E, Gray GA. Highresolution echocardiography in the assessment of cardiac physiology and disease in preclinical models. Exp Physiol. (2013) 98:629-44. doi: 10.1113/expphysiol.2012.068577

59. Branquinho RT, Roy J, Farah C, Garcia GM, Aimond F, Le Guennec J-Y, et al. Biodegradable polymeric nanocapsules prevent cardiotoxicity of antitrypanosomal lychnopholide. Sci Rep. (2017) 7:44998. doi: 10.1038/srep44998

60. Sicard P, Jouitteau T, Andrade-Martins T, Massad A, Rodrigues de Araujo $\mathrm{G}$, David $\mathrm{H}$, et al. Right coronary artery ligation in mice: a novel method to investigate right ventricular dysfunction and biventricular interaction. Am J Physiol Heart Circ Physiol. (2019) 316:H684-92. doi: 10.1152/ajpheart.00573.2018

61. Moreira Souza AC, Grabe-Guimarães A, Cruz JDS, Santos-Miranda A, Farah C, Teixeira Oliveira L, et al. Mechanisms of artemether toxicity on single cardiomyocytes and protective effect of nanoencapsulation. Br J Pharmacol. (2020) 177:4448-63. doi: 10.1111/bph.15186

62. Ader I, Gstalder C, Bouquerel P, Golzio M, Andrieu G, Zalvidea S, et al. Neutralizing S1P inhibits intratumoral hypoxia, induces vascular remodelling and sensitizes to chemotherapy in prostate cancer. Oncotarget. (2015) 6:13803-21. doi: 10.18632/oncotarget.3144

63. Ogier C, Colombo P-E, Bousquet C, Canterel-Thouennon L, Sicard P, Garambois V, et al. Targeting the NRG1/HER3 pathway in tumor cells and cancer-associated fibroblasts with an anti-neuregulin 1 antibody inhibits tumor growth in pre-clinical models of pancreatic cancer. Cancer Lett. (2018) 432:227-36. doi: 10.1016/j.canlet.2018.06.023

64. Graham KC, Wirtzfeld LA, MacKenzie LT, Postenka CO, Groom AC, MacDonald IC, et al. Three-dimensional high-frequency ultrasound imaging for longitudinal evaluation of liver metastases in preclinical models. Cancer Res. (2005) 65:5231-7. doi: 10.1158/0008-5472.CAN-05-0440

65. McVeigh LE, Wijetunga I, Ingram $\mathrm{N}$, Marston $\mathrm{G}$, Prasad $\mathrm{R}$, Markham AF, et al. Development of orthotopic tumour models using ultrasound-guided intrahepatic injection. Sci Rep. (2019) 9:9904. doi: 10.1038/s41598-019-46410-6

66. Pigula M, Huang H-C, Mallidi S, Anbil S, Liu J, Mai Z, et al. Sizedependent tumor response to photodynamic therapy and irinotecan monotherapies revealed by longitudinal ultrasound monitoring in an orthotopic pancreatic cancer model. Photochem Photobiol. (2019) 95:378-86. doi: $10.1111 /$ php. 13016

67. Raes F, Sobilo J, Le Mée M, Rétif S, Natkunarajah S, Lerondel S, et al. High resolution ultrasound and photoacoustic imaging of orthotopic lung cancer in mice: new perspectives for onco-pharmacology. PLoS ONE. (2016) 11:e0153532. doi: 10.1371/journal.pone.0153532

68. Wang S, Zhao Y, Xu Y. Recent advances in applications of multimodal ultrasound-guided photoacoustic imaging technology. Vis Comput Ind Biomed Art. (2020) 3:24. doi: 10.1186/s42492-020-00061-x

69. Mukaddim RA, Rodgers A, Hacker TA, Heinmiller A, Varghese T. Real-time in vivo photoacoustic imaging in the assessment of myocardial dynamics in murine model of myocardial ischemia. Ultrasound Med Biol. (2018) 44:215564. doi: 10.1016/j.ultrasmedbio.2018.05.021

70. Karlas A, Fasoula N-A, Paul-Yuan K, Reber J, Kallmayer M, Bozhko D, et al. Cardiovascular optoacoustics: from mice to men - a review. Photoacoustics. (2019) 14:19-30. doi: 10.1016/j.pacs.2019.03.001

71. David H, Ughetto A, Gaudard P, Plawecki M, Paiyabhroma N, Zub E, et al. Experimental myocardial infarction elicits time-dependent patterns of vascular hypoxia in peripheral organs and in the brain. Front Cardiovasc Med. (2021) 7:615507. doi: 10.3389/fcvm.2020.615507

72. Weber J, Beard PC, Bohndiek SE. Contrast agents for molecular photoacoustic imaging. Nat Methods. (2016) 13:639-50. doi: 10.1038/nmeth.3929 
73. Nishio N, van den Berg NS, Martin BA, van Keulen S, Fakurnejad S, Rosenthal EL, et al. Photoacoustic molecular imaging for the identification of lymph node metastasis in head and neck cancer using an anti-EGFR antibodydye conjugate. J Nucl Med. (2020) 62:648-55. doi: 10.2967/jnumed.120.2 45241

74. Bobo D, Robinson KJ, Islam J, Thurecht KJ, Corrie SR. Nanoparticle-based medicines: a review of FDA-approved materials and clinical trials to date. Pharm Res. (2016) 33:2373-87. doi: 10.1007/s11095-016-1958-5

75. Shi J, Kantoff PW, Wooster R, Farokhzad OC. Cancer nanomedicine: progress, challenges and opportunities. Nat Rev Cancer. (2017) 17:20-37. doi: $10.1038 /$ nrc. 2016.108
Conflict of Interest: The authors declare that the research was conducted in the absence of any commercial or financial relationships that could be construed as a potential conflict of interest.

Copyright $\odot 2021$ Borges, Lages, Sicard, Ferreira and Richard. This is an open-access article distributed under the terms of the Creative Commons Attribution License (CC $B Y)$. The use, distribution or reproduction in other forums is permitted, provided the original author(s) and the copyright owner(s) are credited and that the original publication in this journal is cited, in accordance with accepted academic practice. No use, distribution or reproduction is permitted which does not comply with these terms. 